\title{
Research on the Performance Evaluation of Industry-University-Research Collaborative Innovation Project
}

\author{
Xiaofeng Li \\ Management Science Department of Business School \\ Sichuan University \\ Chengdu City, P.R.C. \\ E-mail: Lixiaofeng@scu.edu.cn
}

\author{
Suying Xiang \\ Management Science Department of Business School \\ Sichuan University \\ Chengdu City, P.R.C. \\ E-mail: xiangsuying@scu.edu.cn
}

\author{
$\mathrm{He} \mathrm{Wu}$ \\ Management Science Department of Business School \\ Sichuan University \\ Chengdu City, P.R.C. \\ E-mail: wuhe@scu.edu.cn \\ Qisheng Chen \\ Management Science Department of Business School \\ Sichuan University \\ Chengdu City, P.R.C. \\ E-mail: chenqisheng@scu.edu.cn
}

\begin{abstract}
To actively promote the deep collaboration and coordinated development between enterprises and universities and research institutes has great importance to building an innovative country. In this paper, on the foundation of the knowledge description of IndustryUniversity-Research Collaborative Innovation, at first, the index system of performance comprehensive evaluation of IU-R collaborative innovation project is established, then the BP neural network model of performance comprehensive evaluation of I-U-R collaborative Innovation project is proposed, and the algorithm of this network model is designed. Theoretical analysis and experimental results show the feasibility and validity of this model. The research work supplies a new way for the performance evaluation of I-U-R collaborative innovation project.
\end{abstract}

Keywords-Collaboration Innovation; Index System; BP Neural Network; Algorithm Design; Performance Evaluation

\section{INTRODUCTION}

As the core of innovation system, I-U-R collaboration is an effective mechanism of achieving the highly integrated of national and regional science and technology, education and industry resources, as well as enhancing independent innovation capability [1]. The imple mentation effect of I-U-R collaboration research becomes an increasing concern of government, society and universities with further promoting of I-U-R collaboration and increasing government funding [2-3]. Knowing how to evaluate the I-U-R collaboration project performance in micro-level and understanding the regional distribution of it has great importance to I-U-R collaboration project, which plays a leading role model and establishes a competition mechanism.

There still exist some problems that the index system is not perfect and the evaluation method is too subjective even though some academics have carried out a few studies on collaborative innovation efficiency and performance evaluation [4-7]. In this case, this paper attempts to make I-U-R collaboration project as research object and completes the establishment of index system from the perspective of the government, and then constructs the BP neural network model and proposes specific algorithm of I-U-R collaboration project performance evaluation. At the end of this paper, an example is used to demonstrate the feasibility and effectiveness of the model, which has a strong practical value.

\section{BP ARTIFICIAL NETWORK ARCHITECTURE AND \\ ALGORITHM}

BP network (Back Propagation), which was proposed by a team of scientists under the leadership of Rumelhart and McCelland in 1986, is a multilayer feed forward network trained by back propagation algorithm as well as one of the most widely used neural network model [8]. This kind of network model has its unique features in dealing with a variety of vague, random, large, lowaccuracy information affairs [9-11]. A standard 3 layers forward propagation neural network is shown in Fig. 1.

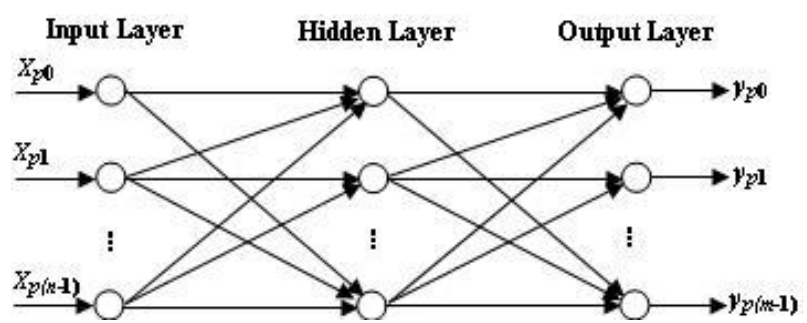

Figure 1. A standard BP artificial neural network.

The first layer consists of $n$ input units. Each of the $n$ input units is connected to each of the $r$ units in the hidden 
layer. The $r$ output units of the hidden layer are all connected to each of the $m$ unit in the output layer.

$\mathrm{BP}$ neural network algorith $\mathrm{m}$ which is also known as error back propagation algorithm has a training process that consists of forward propagation process and back propagation process. The forward propagation process sequentially calculates outputs of every unit from the input layer $\rightarrow$ hidden layer $\rightarrow$ output layer base on the input vector of training samples. The back propagation process sequentially modifies the matrix values $\mathrm{V}$ and $\mathrm{W}$ from the output layer -> hidden layer -> input layer base on the MSE between the actual output of the network and the desired output of the output units. Two processes are alternately repeated until the learning weights converge.

If the BP neural network has $N$ units in each layer, The transfer function is the sigmoid function, $f(x)=\frac{1}{1+e^{-x}}$, the training samples involve $M$ different patterns $\left(X_{p}, Y_{p}\right), P=1,2, \ldots M$. Corresponding the Input sample $P$, let $n e t_{p j}$ represents the input total of unit $j$, let $O_{p j}$ represents the output value, that is

$$
n e t_{p j}=\sum_{j=0}^{N} W_{j i} O_{p j} \quad O_{p j}=f\left(\text { net }_{p j}\right)
$$

The error between input values and output values is as following:

$$
E=\sum E_{p}=\left(\sum\left(d_{p j}-O_{p j}\right)^{2}\right) / 2
$$

The revise connection weights of BP neural network are as following:

$$
\begin{gathered}
W_{j i}=W_{j i}(t)+\eta \delta_{p j} O_{p j} \\
\delta_{p j}=\left[\begin{array}{ll}
f\left(\text { net }_{p j}\right)\left(d_{p j}-O_{p j}\right) & \text { corresponding the output units; } \\
f\left(\text { net }_{p j}\right) \sum \delta_{p k} W_{k j} & \text { corresponding the input units. }
\end{array}\right.
\end{gathered}
$$

where, $\eta$ represents the learning rate, it can increase convergence in speed. Usually there should be an inertial parameter $\alpha$ in the modify weights formula which represents the momentum coefficient. Thus:

$$
W_{j i}=W_{j i}(t)+\eta \delta_{p j} O_{p j}+\alpha\left(W_{j i}(t)-W_{j i}(t-1)\right)
$$

The value of $a$ is a constant, it affects the connection weights of next step. Details of the traditional BP artificial neural network algorithm can be found in the original paper [12].

\section{INDEX SYSTEM EST ABLISHMENT FOR THE I-U-R}

\section{COLLABORATION PROJECT PERFORMANCE EVALUATION}

I-U-R means the mutual collaboration of industries and universities as well as institutions. They can construct an integrated system on the basis of collaboration and outstanding advantages of each other. The integrated system is also an innovation system including complementary advantages and closely linked research and development as well as production [10]. According to systems theory, I-U-R collaboration project performance evaluation includes three basic as pects that are investment, conversion and output. Based on Input - Conversion Output model, this paper decomposed I-U-R collaboration project performance evaluation into three first level indexes that are project investment, project management and project outputs. Associating with the feature of I-U-R collaboration project, the project investment includes three secondary indexes: technical investment, capital investment and talent investment; project management includes two secondary indexes: goal management and organizational management. Based on the view of government, project outputs include four secondary indexes: economic benefits, social benefits, technological benefits and ecological benefits. It is is difficult to measure or obtain ecological benefits' data and the involved content is too less, this paper made the ecological benefits as a secondary indicator of secondary indicator. That's to say; finally the project outputs includes three secondary indexes: economic benefits, social benefits and technological

\begin{tabular}{|c|c|c|}
\hline $\begin{array}{c}\text { Evaluation } \\
\text { objective }\end{array}$ & $\begin{array}{c}\text { First Level } \\
\text { Indexes }\end{array}$ & Secondary Indexes \\
\hline \multirow{8}{*}{$\begin{array}{c}\text { I-U-R } \\
\text { collaboration } \\
\text { project } \\
\text { performance }\end{array}$} & \multirow{3}{*}{$\begin{array}{c}\text { Project } \\
\text { Investment }\end{array}$} & Technical Investment $u_{1}$ \\
\hline & & Capital Investment $u_{2}$ \\
\hline & & Talent Investment $u_{3}$ \\
\hline & \multirow{2}{*}{$\begin{array}{c}\text { Project } \\
\text { Management }\end{array}$} & Goal Management $u_{4}$ \\
\hline & & Organizational Management $u_{5}$ \\
\hline & \multirow{3}{*}{$\begin{array}{l}\text { Project } \\
\text { Outputs }\end{array}$} & Economic Benefits $u_{6}$ \\
\hline & & Social Benefits $u_{7}$ \\
\hline & & Technological Benefits $u_{8}$ \\
\hline
\end{tabular}
benefits. The final index system of the I-U-R collaboration project performance evaluation is shown in Table I.

TABLE I. INDEX SYSTEM OF THE I-U-R COLLABORATION PROJECT PERFORMANCE EVALUATION

IV. THE BP NEURAL NETWORK MODEL FOR I-U-R COLLABORATION PROJECT PERFORMANCE EVALUATION

\section{A. The Assignment Method of Evaluation Indexes}

Secondary indexes of I-U-R collaboration project performance evaluation include qualitative indexes and quantitative indexes. In this paper, the assignment method of secondary indexes is fuzzy statistics. Firstly determine the membership function, and then determine the evaluation value. Assuming the evaluation set of I-U-R collaboration project performance which has descending reviews is:

$\mathrm{V}=\{$ excellent, good, mediu $\mathrm{m}$, qualified, unqualified $\} \equiv$ $\left\{A_{1}, A_{2}, \cdots, A_{6}\right\}=\{1,2,3,4,5,6\}$

Fuzzy statistics lets the experts involved in the assessment (n experts in total) give their revie ws according to the pre-defined evaluation set $\mathrm{V}$. Then the statistics frequency meets for every review At $(t=1, \ldots, 6)$ for every secondary indexes $u_{i}$. Denoted as

$$
\mu_{i}^{(t)}=\frac{m_{i t}}{n}
$$

$\mu_{i}^{(t)}$ represents the membership degree between evaluation index $u_{i}$ and review $\mathrm{A}_{t}$; Denoted again:

$$
R_{i}=\mu_{i}^{(1)} / A_{1}+\cdots+\mu_{i}^{(6)} / A_{6}
$$
index $u_{i}$ 


\section{B. The Designed Performance Evaluation Model}

Researchers designed performance evaluation model as three parts according to the BP neural network which is introduced in part 2 of this paper.

1) Input layer: According to the index system of I-U-R collaboration project performance evaluation, this paper made the secondary indexes (as shown in Table 1) as the input layer' units ( 8 in total). Then assigned values for the indexes and made the values as training samples of BP neural network.

2) Hidden layer: As for the selection of the hidden layer researchers refer to the literature [13] to propose BP neural network adaptive learning algorithm. Setting enough hidden layer nodes at first to make the network self-regulated learning and ultimately got the right size of the hidden layer nodes.

3) Output layer: The process of the I-U-R collaboration project performance evaluation is from qualitative to quantitative and then to qualitative again. Firstly transformed qualitative into quantitative output by BP neural network, and then made comprehensive evaluation of I-U-R collaboration project performance.Researchers divided the evaluation into 6 grades: excellent, good, medium, qualified, unqualified. And the output vector is represented by $(1,0,0,0$, $0,0),(0,1,0,0,0,0),(0,0,1,0,0$, $0),(0,0,0,1,0,0),(0,0,0,0,1,0),(0$, $0,0,0,0,1)$ in the BP neural network. Therefore, the number of output layer nodes in the neural network is 6.

\section{Performance Evaluation Algorithm}

A BP neural network according to the above model structure design is formed. The basic steps for I-U-R collaboration project performance evaluation are as are as follows:

- Collect the training samples of different collaborative innovation project and process data according to the index system of the I-U-R collaboration project performance evaluation.

- Construct BP neural network, take 8 input layer nodes and 6 output layer nodes of hidden layer, and then initialize the network.

- Input the data which is processed in Step (1) to the BP neural network, and then determine the weight and the threshold value between the layers according to adaptive learning algorith $m$ of the BP neural network.

- Collect index values of the evaluated collaborative innovation project and then process data.

- Input the processed data to the BP neural network and calculate the output.

- Determine the assessment degree of the collaborative innovation project to be evaluated according to the output and the evaluation set.

\section{APPLICATION EXAMPLE}

Researchers conduct the method mentioned above on I-U-R collaboration project performance evaluation. Before the evaluation, researchers collect the classic training samples (the index values of different collaborative innovation project) base on the index system of I-U-R collaboration project performance evaluation. Researchers collect 10 indexes values of IU-R collaboration project in Chengdu City and then process the data whose short names and codes are: environmental friendly polypropylene film $\left(\mathrm{Q}_{1}\right)$, oil filter $\left(Q_{2}\right)$, green ceramic manufacturing process $\left(Q_{3}\right)$, new water heater $\left(Q_{4}\right)$, emergency escape device $\left(Q_{5}\right)$, high quality fire-retardant material $\left(\mathrm{Q}_{6}\right)$, fast compiler $\left(Q_{7}\right)$, centrifugal extractor $\left(Q_{8}\right)$, miniature weighing sensor $\left(Q_{9}\right)$, numerical control gumming machine $\left(\mathrm{Q}_{10}\right)$. Researchers construct a BP neural network as 817-6 (8 input layer units, 17 hidden layer units, 6 output layer units) and then initialize the netw ork (error upper bound $\varepsilon=0.0002$, learning rate $\eta=0.5$, inertial parameter $\mathrm{a}=0.1$ ). Input the processed data to the BP neural network; train the network according to the adaptive learning algorithm of the BP neural netw ork to get a trained 8-11-6 BP network. The netw ork inference output of the training samples is shown in Table 1.Researchers can see from the Table that the network inference output and the actual situation match very well which represents that the BP neural network of IU-R collaboration project performance evaluation has feasibility and effectiveness.

Putting the collected index values of a Chengdu enterprise's I-U-R collaboration project (research and development of solar energy conversion device, code Q) into the trained BP neural network and calculate the output which is shown in Table 2.Researchers can see from the evaluation result that the performance degree of the collaborative innovation project (code Q) is medium.

\section{CONCLUSION}

This paper makes full use of self-organizing learning ability of BP neural network to construct I-U-R collaboration project performance evaluation model. This model not only works well in the evaluation of I-U-R collaboration project performance but also effectively eliminates the human factors and fuzzy randomness effects which are made by manual assessments as well as ensures the objectivity and accuracy of the evaluation results. This provides a feasible approach for the performance evaluation of I-U-R collaboration project.

\section{ACKNOWLEDGEMENT}

This research was supported by the Soft Science Research Project of Chengdu (2014-RK00-00014-ZF) and Soft Science Research Plan Project of Sichuan Province (2015ZR0016).

\section{REFERENCES}

[1] Zheng Zhou, Linna Yin and Bing, "Research on the IndustryUniversity-Research Driving Mechanism of China ", Soft Science, pp.12-19,July 2013.

[2] Jinfu Wang, Yingying Zhang and Shibin Su, "Research on the Industry-University-Research Driving Mechanism", Science \& Technology Progress and Policy, vol.30, pp. 2127-2130, December. 2013. 
[3] Xia Fan, Liming Chen and Wei Liu, "Empirical Research on Innovation Effects of University Industry Collative Innovation Based on Propensity Score Matching Method", Science of Science and Management of S. \& T, pp.63-69, February 2013.

[4] Yan Huo, "Research on the Index System and Evaluation Method of Industry-University-Research Collaboration". Science \& Technology Progress and Policy, pp.79-83, June 2014.

[5] Jing Cao, Decheng Fan and Xiaoxu Tang, "Research on the Performance Evaluation of Industry-University-Research and Technology Innovation", Science \& Technology Progress and Policy, pp.114-118, July 2010.

[6] Dingding Xiao and Guilong Zhu, "Empirical Research on IndustryUniversity-Research Cooperative Innovation Efficiency and Influencing Factors", Science Research Management, pp.11-19, January 2013.

[7] Decheng Fan and Xiaoxu Tang, "Research on the Performance Evaluation of Industry-University-Research and Technology
Innovation in Different Province of China", Science of Science and Management of S. \& T, pp.66-70, January 2009.

[8] Hagan M T, Men haj M B., "Training Feed Forward Networks with Marquetry Algorithm", IEEE Trans. on Neural Networks, pp.989-993, vol.5,May 1994.

[9] Hecht-Nielsen R, "Theory of Back Propagation Neural Network", Proc, of IJCNN, pp.593-603, 1989.

[10] Liqun Han, "The Theory, Design and Application of Artificial Neural Network", Beijing: Chemical Industry Press, pp.17-29, 2002.

[11] Naiyao Zhang and Pingfan Yan, "Neural Networks and Fuzzy Control", Beijing: Tsinghua University Press, pp.7-21, 1998.

[12] Farlow,S.J. , "Self-organizing Method in Modeling", Marcel Dekker. New York, 1996.

[13] Xiaofeng Li and Jiuping Xu, "The Establishment and Application of BP Neural Network Adaptive Learning Algorithm", Systems Engineering Theory \& Practice, vol.24, pp.1-8,May 2004.

TABLE II. NETWORK INFERENCE OUTPUT

\begin{tabular}{|c|c|c|c|c|}
\hline $\begin{array}{l}\text { Project } \\
\text { Code }\end{array}$ & $\begin{array}{l}\text { Actual Situation of } \\
\text { Collaborative } \\
\text { Innovation Project } \\
\text { Performance }\end{array}$ & $\begin{array}{c}\text { Training Sample } \\
\text { Output }\end{array}$ & Network Inference output & $\begin{array}{l}\text { Model } \\
\text { e valuation } \\
\text { results }\end{array}$ \\
\hline $\mathrm{Q}_{1}$ & medium & $(0,0,0,1,0,0)$ & $\left(\begin{array}{lllllll}0.0021 & 0.0041 & 0.0368 & 1.0068 & 0.0223 & -0.0383\end{array}\right)$ & medium \\
\hline $\mathrm{Q}_{2}$ & unqualified & $(0,0,0,0,0,1)$ & $(0.00610 .00520 .00750 .00820 .14750 .9998)$ & unqualified \\
\hline $\mathrm{Q}_{3}$ & medium & $(0,0,1,0,0,0)$ & $(0.0017-0.00251 .02350 .006810 .0325-0.07568)$ & medium \\
\hline $\mathrm{Q}_{4}$ & excellent & $(1,0,0,0,0,0)$ & $(0.99970 .12010 .02457-0.097630 .01760 .0342)$ & excellent \\
\hline $\mathrm{Q}_{5}$ & excellent & $(1,0,0,0,0,0)$ & $(0.99990 .00380 .0007-0.06430 .15470 .0089)$ & excellent \\
\hline $\mathrm{Q}_{6}$ & medium & $(0,0,1,0,0,0)$ & $(0.00870 .00751 .0010 .09100 .12690 .0021)$ & medium \\
\hline $\mathrm{Q}_{7}$ & qualified & $(0,0,0,0,1,0)$ & $(0.0810 .25890 .03520 .00370 .9999-0.2551)$ & qualified \\
\hline $\mathrm{Q}_{8}$ & good & $(0,1,0,0,0,0)$ & $(0.04531 .00090 .00410 .0086-0.21300 .0025)$ & good \\
\hline $\mathrm{Q}_{9}$ & excellent & $(1,0,0,0,0,0)$ & $(1.00030 .21490 .00870 .0914-0.38210 .0046)$ & excellent \\
\hline $\mathrm{Q}_{10}$ & good & $(0,1,0,0,0,0)$ & $(0.23160 .99870 .18740 .13230 .00960 .0157)$ & good \\
\hline Q & \multicolumn{2}{|c|}{ Forecast } & $\left(\begin{array}{llllll}0.0036 & 0.0378 & 0.9978 & 0.0093 & 0.0167 & 0.0251\end{array}\right)$ & medium \\
\hline
\end{tabular}

